\title{
On weighted Iyengar-type inequalities for conformable fractional integrals
}

\author{
Mehmet Zeki Sarikaya $^{1} \cdot$ Hatice Yaldiz $^{1} \cdot$ Hüseyin Budak ${ }^{1}$
}

Received: 23 September 2016/Accepted: 30 August 2017/Published online: 11 September 2017

(c) The Author(s) 2017. This article is an open access publication

\begin{abstract}
In this study, we establish some new weighted Iyengar-type integral inequalities using fractional Steffensen's inequality for conformable fractional integral. The results presented here would provide generalizations of those given in earlier works.
\end{abstract}

Keywords Trapezoid inequality · Iyengar inequality ·

Weighted quadrature rule $\cdot$ Conformable fractional integral

Mathematics Subject Classification 26D15 - 26A33 . $26 \mathrm{~A} 42$

\section{Introduction}

The presence of a differentiable mappings was introduced in 1938 for the first time and the results were announced by Iyengar. In recent years, Iyengar-type integral inequalities have been studied by several authors such as $[5,6,9,10,12]$. In this paper, we concentrate on Iyengartype integral inequalities and present some generalizations. Section 2 consists of conformable integrals, derivatives, and some properties of them. Steffensen's inequality for

Hatice Yaldiz

yaldizhatice@gmail.com

Mehmet Zeki Sarikaya

sarikayamz@gmail.com

Hüseyin Budak

hsyn.budak@gmail.com

1 Department of Mathematics, Faculty of Science and Arts, Düzce University, Düzce, Turkey conformable fractional integral is also given. Finally, in Sect. 3, my means of Steffensen's inequality, we obtain some main interesting results. We first establish two new identities for conformable integral involving some moments. Generalizing this, using these equalities and considering fractional Steffensen's inequality for conformable fractional integrals, we get a new version of weighted Iyengar-type integral inequalities for conformable integrals.

The results presented here will provide generalizations of those given in earlier works. Iyengar proved a useful inequality which gives an upper bound for the approximation of the integral average by the mean of the values of mapping at end points of the interval that is given below (see [7] or [11, p.471]).

Theorem 1.1 Let $f$ be a differentiable function on $(a, b)$ and assume that there is a constant $M>0$, such that $\left|f^{\prime}(x)\right| \leq M, \forall x \in(a, b)$. Then, we have

$$
\begin{aligned}
& \left|\int_{a}^{b} f(x) \mathrm{d} x-(b-a) \frac{f(a)+f(b)}{2}\right| \leq \frac{M(b-a)^{2}}{4} \\
& \quad-\frac{1}{4 M}(f(b)-f(a))^{2} .
\end{aligned}
$$

In [3], Agarwal and Dragomir proved the following generalization of Theorem 1.1.

Theorem 1.2 Let $f: I \subseteq \mathbb{R} \rightarrow \mathbb{R}$ be a differentiable mapping on $I^{\circ}$ (the interior of $I$ ), $[a, b] \subset I^{\circ}$ with $a<b$. Assume that $M=\sup _{x \in[a, b]} f^{\prime}(x)<\infty$ and $m=$ $\inf _{x \in[a, b]} f^{\prime}(x)>-\infty$. If $M>m$ and $f^{\prime}$ is integrable on $[a, b]$, then the following inequality holds: 


$$
\begin{aligned}
& \left|\int_{a}^{b} f(x) \mathrm{d} x-(b-a) \frac{f(a)+f(b)}{2}\right| \\
& \quad \leq \frac{[M(b-a)-f(b)+f(a)][f(b)-f(a)-m(b-a)]}{2(M-m)} .
\end{aligned}
$$

Using a classical Steffensen's inequality, Liu proved a generalization of the above result involving weighted integrals in terms of bounds involving the first derivative of the function.

The following Lemmas and Theorem was given by him in [9] (or [5]).

Lemma 1.1 Let $f: I \subseteq \mathbb{R} \rightarrow \mathbb{R}$ be a differentiable mapping on $I^{\circ}$ (the interior of $I$ ) and $[a, b] \subset I^{\circ}$ with $M=\sup _{x \in[a, b]} f^{\prime}(x)<\infty, m=\inf _{x \in[a, b]} f^{\prime}(x)>-\infty$, and $M>m$. Assume that $w(x) \geq 0$ for all $x \in[a, b] \mu=$ $\int_{a}^{b} w(x) \mathrm{d} x<\infty$ and $v=\int_{a}^{b} x w(x) \mathrm{d} x<\infty$ be the zeroth and first moments of $w($.$) on [a, b]$. If $f^{\prime}$ is integrable on $[a, b]$, then the following inequality holds:

$$
\begin{aligned}
{[M-m]\left[Q_{b}-(b-\lambda) P_{b}\right] } & \leq \int_{a}^{b} w(x) f(x) \mathrm{d} x \\
-\mu(f(a)-m a)-m v & \leq[M-m]\left[Q_{a}-(\lambda+a) P_{a}+\lambda \mu\right] .
\end{aligned}
$$

where $\quad P_{a}=\int_{a}^{a+\lambda} w(x) \mathrm{d} x, \quad Q_{b}=\int_{b-\lambda}^{b} w(x) \mathrm{d} x \quad$ and $\lambda=\frac{b-a}{M-m}\left(\frac{f(b)-f(a)}{b-a}-m\right)$.

Lemma 1.2 Let the conditions be as in Lemma 1.1, then the following inequality holds:

$$
\begin{aligned}
& {[M-m]\left[Q_{b}-(\lambda-b) P_{b}-\lambda \mu\right] \leq \int_{a}^{b} w(x) f(x) \mathrm{d} x} \\
& -\mu(f(b)-m b)-m v \leq[M-m]\left[Q_{a}-(\lambda+a) P_{a}\right] .
\end{aligned}
$$

Let the condition of Lemma 1.1 and 1.2 be maintained. Then, the following inequality holds:

$$
\begin{aligned}
& {[M-m]\left[Q_{b}-(b-\lambda) P_{b}-\frac{\lambda}{2} \mu\right] \leq \int_{a}^{b} w(x) f(x) \mathrm{d} x} \\
& \quad-\frac{\mu}{2}(f(a)+f(b)-m(a+b)) \\
& \quad-m v \leq[M-m]\left[Q_{a}-(\lambda+a) P_{a}+\frac{\lambda}{2} \mu\right] .
\end{aligned}
$$

Now, we will introduce the conformable integral and derivative:

\section{Definition and properties of conformable fractional derivative and integral}

The following definitions and theorems with respect to conformable fractional derivative and integral were referred in (see, [1, 2, 4, 8]).

Definition 2.1 (Conformable fractional derivative) Given a function $f:[0, \infty) \rightarrow \mathbb{R}$. Then, the "conformable fractional derivative" of $f$ of order $\alpha$ is defined by

$D_{\alpha}(f)(t)=\lim _{\varepsilon \rightarrow 0} \frac{f\left(t+\varepsilon t^{1-\alpha}\right)-f(t)}{\varepsilon}$

for all $t>0, \alpha \in(0,1)$.If $f$ is $\alpha$-differentiable in some $(0, a), \alpha>0, \lim _{t \rightarrow 0^{+}} f^{(\alpha)}(t)$ exist, then define

$f^{(\alpha)}(0)=\lim _{t \rightarrow 0^{+}} f^{(\alpha)}(t)$.

We can write $f^{(\alpha)}(t)$ for $D_{\alpha}(f)(t)$ to denote the conformable fractional derivatives of $f$ of order $\alpha$. In addition, if the conformable fractional derivative of $f$ of order $\alpha$ exists, then we simply say $f$ is $\alpha$-differentiable.

Theorem 2.1 Let $\alpha \in(0,1]$ and $f$ and $g$ be $\alpha$-differentiable at a point $t>0$. Then

(i) $D_{\alpha}(a f+b g)=a D_{\alpha}(f)+b D_{\alpha}(g)$, for all $a, b \in \mathbb{R}$,

(ii) $D_{\alpha}(\lambda)=0$,for all constant functions $f(t)=\lambda$,

(iii) $D_{\alpha}(f g)=f D_{\alpha}(g)+g D_{\alpha}(f)$,

(iv) $D_{\alpha}\left(\frac{f}{g}\right)=\frac{f D_{\alpha}(g)-g D_{\alpha}(f)}{g^{2}}$.

If $f$ is differentiable, then

$D_{\alpha}(f)(t)=t^{1-\alpha} \frac{\mathrm{d} f}{\mathrm{~d} t}(t)$.

Definition 2.2 (Conformable fractional integral) Let $\alpha \in$ $(0,1]$ and $0 \leq a<b$. A function $f:[a, b] \rightarrow \mathbb{R}$ is $\alpha$-fractional integrable on $[a, b]$ if the integral

$\int_{a}^{b} f(x) \mathrm{d}_{\alpha} x:=\int_{a}^{b} f(x) x^{\alpha-1} \mathrm{~d} x$

exists and is finite. All $\alpha$-fractional integrable on $[a, b]$ is indicated by $L_{\alpha}^{1}([a, b])$.

Remark 2.1

$I_{\alpha}^{a}(f)(t)=I_{1}^{a}\left(t^{\alpha-1} f\right)=\int_{a}^{t} \frac{f(x)}{x^{1-\alpha}} \mathrm{d} x$,

where the integral is the usual Riemann improper integral, and $\alpha \in(0,1]$.

Theorem 2.2 Let $f:(a, b) \rightarrow \mathbb{R}$ be differentiable and $0<\alpha \leq 1$. Then, for all $t>a$, we have 
$I_{\alpha}^{a} D_{\alpha}^{a} f(t)=f(t)-f(a)$.

Theorem 2.3 (Integration by parts) Let $f, g:[a, b] \rightarrow \mathbb{R}$ be two functions, such that $f g$ is differentiable. Then

$\int_{a}^{b} f(x) D_{\alpha}^{a}(g)(x) \mathrm{d}_{\alpha} x=\left.f g\right|_{a} ^{b}-\int_{a}^{b} g(x) D_{\alpha}^{a}(f)(x) \mathrm{d}_{\alpha} x$.

The following theorem gave by Anderson [4].

Theorem 2.4 (Fractional Steffensen's inequality) Let $\alpha \in(0,1]$ and $a$ and $b$ be real numbers, such that $0 \leq a<b$. Let $h:[a, b] \rightarrow[0, \infty)$ and $g:[a, b] \rightarrow[0,1]$ be $\alpha$-fractional integrable functions on $[a, b]$ with $h$ is decreasing. Then

$\int_{b-\ell}^{b} h(x) \mathrm{d}_{\alpha} x \leq \int_{a}^{b} h(x) g(x) \mathrm{d}_{\alpha} x \leq \int_{a}^{a+\ell} h(x) \mathrm{d}_{\alpha} x$,

where $\ell:=\frac{\alpha(b-a)}{b^{\alpha}-a^{\alpha}} \int_{a}^{b} g(x) \mathrm{d}_{\alpha} x$.

\section{Weighted trapezoidal inequality for conformable fractional integrals}

Some definitions are required to simplify the subsequent work.

Definition 3.1 Let $w(x)$ be a positive conformable integrable function on $[a, b]$ and $\alpha \in(0,1]$. Let $\mu$ and $v$ be its zeroth and first moments about zero, so that

$\mu_{\alpha}=\frac{1}{\alpha} \int_{a}^{b} w(x) \mathrm{d}_{\alpha} x<\infty$,

and

$v_{\alpha}=\frac{1}{\alpha} \int_{a}^{b} x^{\alpha} w(x) \mathrm{d}_{\alpha} x<\infty$.

Definition 3.2 $P$ and $Q$ will be used to denote the zeroth and first moments of $w(x)$ over a subinterval $[a, b]$. In particular, for $\ell>0$, the subscript $a$ and $b$ will be used to indicate the intervals $[a, a+\ell]$ and $[b-\ell, b]$, respectively. Thus, for example

$$
\begin{aligned}
& P_{\alpha}(a, \ell):=\frac{1}{\alpha} \int_{a}^{a+l} w(x) \mathrm{d}_{\alpha} x, \\
& Q_{\alpha}(a, \ell):=\frac{1}{\alpha} \int_{a}^{a+\ell} x^{\alpha} w(x) \mathrm{d}_{\alpha} x,
\end{aligned}
$$

$P_{\alpha}(\ell, b):=\frac{1}{\alpha} \int_{b-\ell}^{b} w(x) \mathrm{d}_{\alpha} x$,

and

$Q_{\alpha}(\ell, b):=\frac{1}{\alpha} \int_{b-\ell}^{b} x^{\alpha} w(x) \mathrm{d}_{\alpha} x$.

Fractional Steffensen's inequality (2.7) will now be used to obtain inequalities for conformable fractional integrals to give weighted trapezoidal-type quadrature rules. First, the following lemmas will need to be proved by the use of the Fractional Steffensen's inequality.

Lemma 3.1 Let $\alpha \in(0,1]$ and $a, b \in \mathbb{R}$ with $0 \leq a<b$, $f:[a, b] \rightarrow \mathbb{R}$ be a conformable differentiable with $M=\sup _{x \in[a, b]} D_{\alpha} f(x)<\infty, \quad m=\inf _{x \in[a, b]} D_{\alpha} f(x)>-\infty$ and $M>m$. Assume that $w(x) \geq 0$ for all $x \in[a, b]$ and $v=\frac{1}{\alpha} \int_{a}^{b} x^{\alpha} w(x) \mathrm{d}_{\alpha} x<\infty$ be the first moment of $w($.$) on$ $[a, b]$. If $D_{\alpha} f$ is $\alpha$-fractional integrable on $[a, b]$, then the following inequalities hold:

$$
\begin{aligned}
& {[M-m]\left[Q_{\alpha}(\ell, b)-(b-\ell)^{\alpha} P_{\alpha}(\ell, b)\right]} \\
& \leq \int_{a}^{b} w(x) f(x) \mathrm{d}_{\alpha} x-\mu_{\alpha}\left(\alpha f(a)-m a^{\alpha}\right)-m v_{\alpha} \\
& \leq[M-m]\left[Q_{\alpha}(a, \ell)-(a+\ell)^{\alpha} P_{\alpha}(a, \ell)+\left[(a+\ell)^{\alpha}-a^{\alpha}\right] \mu_{\alpha}\right],
\end{aligned}
$$

where $P_{\alpha}$ and $Q_{\alpha}$ are as described in Definition 3.2.

Proof Let $h_{b}(x)=\int_{x}^{b} w(u) \mathrm{d}_{\alpha} u$ and $g(x)=\frac{D_{\alpha} f(x)-m}{M-m}$. Then, from inequality (2.7), we get

$L_{b} \leq I_{b} \leq U_{b}$,

where

$L_{b}=(M-m) \int_{b-\ell}^{b} h_{b}(x) \mathrm{d}_{\alpha} x$,

$I_{b}=\int_{a}^{b} h_{b}(x)\left(D_{\alpha} f(x)-m\right) \mathrm{d}_{\alpha} x$,

and

$U_{b}=(M-m) \int_{a}^{a+\ell} h_{b}(x) \mathrm{d}_{\alpha} x$.

Now, an integration by parts and change of order formula gives 


$$
\begin{aligned}
I_{b}= & \int_{a}^{b}\left(\int_{x}^{b} w(u) \mathrm{d}_{\alpha} u\right) D_{\alpha} f(x) \mathrm{d}_{\alpha} x \\
& -m \int_{a}^{b}\left(\int_{x}^{b} w(u) \mathrm{d}_{\alpha} u\right) \mathrm{d}_{\alpha} x \\
= & \left(\int_{x}^{b} w(u) \mathrm{d}_{\alpha} u\right) f(x)+\int_{a}^{b} w(x) f(x) \mathrm{d}_{\alpha} x \\
& -m \int_{a}^{b} w(u)\left(\int_{a}^{u} \mathrm{~d}_{\alpha} u\right)_{a}^{b} w \int_{a}^{b} w(x) f(x) \mathrm{d}_{\alpha} x .
\end{aligned}
$$

In addition

$$
\ell=\frac{\alpha(b-a)}{\left(b^{\alpha}-a^{\alpha}\right)(M-m)} \int_{a}^{b}\left(D_{\alpha} f(x)-m\right) \mathrm{d}_{\alpha} x .
$$

It should be noted that $0<\ell \leq b-a$.

For the lower bound $L_{b}$, a change of order of integration gives

$$
\begin{aligned}
\frac{L_{b}}{M-m} & =\int_{b-\ell}^{b}\left(\int_{x}^{b} w(u) \mathrm{d}_{\alpha} u\right) \mathrm{d}_{\alpha} x \\
& =Q_{\alpha}(\ell, b)-(b-\ell)^{\alpha} P_{\alpha}(\ell, b),
\end{aligned}
$$

where $P_{\alpha}(\ell, b)$ and $Q_{\alpha}(\ell, b)$ are described in Definition 3.2.

Similarly, the upper bound $U_{b}$ may be obtained through a change of order of integration to give

$$
\begin{aligned}
\frac{U_{b}}{M-m}= & \int_{a}^{a+\ell}\left(\int_{x}^{b} w(u) \mathrm{d}_{\alpha} u\right) \mathrm{d}_{\alpha} x \\
= & \int_{a}^{b} \int_{x}^{b} w(u) \mathrm{d}_{\alpha} u \mathrm{~d}_{\alpha} x-\int_{a+\ell}^{b} \int_{x}^{b} w(u) \mathrm{d}_{\alpha} u \mathrm{~d}_{\alpha} x \\
= & \int_{a}^{b} w(u)\left(\int_{a}^{u} \mathrm{~d}_{\alpha} x\right) \mathrm{d}_{\alpha} u \\
& -\int_{a+\ell}^{b} w(u)\left(\int_{a+\ell}^{u} \mathrm{~d}_{\alpha} x\right) \mathrm{d}_{\alpha} u \\
= & Q_{\alpha}(a, \ell)-(a+\ell)^{\alpha} P_{\alpha}(a, \ell)+\left[(a+\ell)^{\alpha}-a^{\alpha}\right] \frac{\mu_{\alpha}}{2},
\end{aligned}
$$

where $P_{\alpha}(a, \ell)$ and $Q_{\alpha}(a, \ell)$ are described in Definition 3.2, and $v_{\alpha}$ is the zeroth moment of $w(x)$ on $[a, b]$.

Using (3.8)-(3.15), the lemma is thus proved.
Lemma 3.2 Let the conditions be as in Lemma 3.1, then the following inequalities hold:

$$
\begin{array}{r}
{[M-m]\left[Q_{\alpha}(\ell, b)-(b-\ell)^{\alpha} P_{\alpha}(\ell, b)+\left[(b-\ell)^{\alpha}-b^{\alpha}\right] \mu_{\alpha}\right]} \\
\leq \int_{a}^{b} w(x) f(x) \mathrm{d}_{\alpha} x-\mu_{\alpha}\left(\alpha f(b)-m b^{\alpha}\right)-m v_{\alpha} \\
\quad \leq[M-m]\left[Q_{\alpha}(a, \ell)-(a+\ell)^{\alpha} P_{\alpha}(a, \ell)\right] .
\end{array}
$$

where $P_{\alpha}$ and $Q_{\alpha}$ are as described in Definition 3.2.

Proof The proof follows along similar lines to that of Lemma 3.1. Let $h_{a}(x)=-\int_{a}^{x} w(u) \mathrm{d}_{\alpha} u$ and $g(x)=$ $\frac{D_{\alpha} f(x)-m}{M-m}$. Then, from inequality (2.7)

$L_{a} \leq I_{a} \leq U_{a}$,

where

$I_{a}=\int_{a}^{b} h_{a}(x)\left(D_{\alpha} f(x)-m\right) \mathrm{d}_{\alpha} x$

$L_{a}=(M-m) \int_{b-\ell}^{b} h_{a}(x) \mathrm{d}_{\alpha} x$

and

$U_{a}=(M-m) \int_{a}^{a+\ell} h_{a}(x) \mathrm{d}_{\alpha} x$.

Now, a straight forward integration by parts yields

$$
\begin{aligned}
I_{a} & =\int_{a}^{b} h_{a}(x)\left(D_{\alpha} f(x)-m\right) \mathrm{d}_{\alpha} x \\
& =\int_{a}^{b} w(x) f(x) \mathrm{d}_{\alpha} x-\mu_{\alpha}\left(\alpha f(b)-m b^{\alpha}\right)-m v_{\alpha} .
\end{aligned}
$$

Furthermore, an interchange of the order of integration and simplification of results yields

$$
\begin{aligned}
L_{a} & =(M-m) \int_{b-\ell}^{b} h_{a}(x) \mathrm{d}_{\alpha} x \\
& =(M-m)\left[Q_{\alpha}(\ell, b)-(b-\ell)^{\alpha} P_{\alpha}(\ell, b)+\left[(b-\ell)^{\alpha}-b^{\alpha}\right] \mu_{\alpha}\right],
\end{aligned}
$$

and

$$
U_{a}=(M-m) \int_{a}^{a+\ell} h_{a}(x) \mathrm{d}_{\alpha} x=Q_{\alpha}(a, \ell)-(a+\ell)^{\alpha} P_{\alpha}(a, \ell) .
$$


Hence, using (3.17)-(3.20), the lemma is thus proved.

Theorem 3.1 Let the condition of Lemmas 3.1 and 3.2 be maintained. Then, the following inequality holds:

$$
\begin{aligned}
& {[M-m]\left[Q_{\alpha}(\ell, b)-(b-\ell)^{\alpha} P_{\alpha}(\ell, b)+\left[(b-\ell)^{\alpha}-b^{\alpha}\right] \frac{\mu_{\alpha}}{2}\right]} \\
& \quad \leq \int_{a}^{b} w(x) f(x) \mathrm{d}_{\alpha} x-\mu_{\alpha}\left[\alpha \frac{f(a)+f(b)}{2}-m \frac{a^{\alpha}+b^{\alpha}}{2}\right]-m v_{\alpha} \\
& \quad \leq[M-m]\left[Q_{\alpha}(a, \ell)-(a+\ell)^{\alpha} P_{\alpha}(a, \ell)+\left[(a+\ell)^{\alpha}-a^{\alpha}\right] \frac{\mu_{\alpha}}{2}\right],
\end{aligned}
$$

where the $P_{\alpha} s$ and $Q_{\alpha} s$ are as defined in Definition 3.2.

Proof Adding (3.7) and (3.16), and resulting inequalities divided by 2 . This completes the proof.

Corollary 3.1 Let the conditions be as in the previous lemmas and theorem of this section. Then

$$
\begin{aligned}
& \left|\int_{a}^{b} w(x) f(x) \mathrm{d}_{\alpha} x-\mu_{\alpha}\left[\alpha \frac{f(a)+f(b)}{2}-m \frac{a^{\alpha}+b^{\alpha}}{2}\right]-m v_{\alpha}\right| \\
& \leq \frac{b^{\alpha}-a^{\alpha}}{2} \mu_{\alpha} .
\end{aligned}
$$

Proof The corollary follows readily from (3.21) on noting that:

$$
\begin{aligned}
& Q_{\alpha}(\ell, b)=\frac{1}{\alpha} \int_{b-\ell}^{b} x^{\alpha} w(x) \mathrm{d}_{\alpha} x \geq \frac{(b-\ell)^{\alpha}}{\alpha} \int_{b-\ell}^{b} w(x) \mathrm{d}_{\alpha} x, \\
& Q_{\alpha}(a, \ell)=\frac{1}{\alpha} \int_{a}^{a+\ell} x^{\alpha} w(x) \mathrm{d}_{\alpha} x \leq \frac{(a+\ell)^{\alpha}}{\alpha} \int_{a}^{a+\ell} w(x) \mathrm{d}_{\alpha} x,
\end{aligned}
$$

and

$$
\begin{array}{ll}
(b-\ell)^{\alpha}-b^{\alpha} \geq a^{\alpha}-b^{\alpha}, & a<b-\ell<b, \\
(a+\ell)^{\alpha}-a^{\alpha} \leq b^{\alpha}-a^{\alpha}, & a<a+\ell<b .
\end{array}
$$

Hence, this completes the proof.

Remark 3.1 If we take $\alpha=1$ in Lemma 3.1, then inequality (3.7) reduces inequality (1.2).

Remark 3.2 If we take $\alpha=1$ in Lemma 3.2, then inequality (3.16) reduces inequality (1.3).
Remark 3.3 If we take $\alpha=1$ in Theorem 3.1, then inequality (3.21) reduces inequality (1.4).

Open Access This article is distributed under the terms of the Creative Commons Attribution 4.0 International License (http://crea tivecommons.org/licenses/by/4.0/), which permits unrestricted use, distribution, and reproduction in any medium, provided you give appropriate credit to the original author(s) and the source, provide a link to the Creative Commons license, and indicate if changes were made.

Publisher's Note Springer Nature remains neutral with regard to jurisdictional claims in published maps and institutional affiliations.

\section{References}

1. Abu Hammad, M., Khalil, R.: Conformable fractional heat differential equations. Int. J. Differ. Equ. Appl. 13(3), 177-183 (2014)

2. Abu Hammad, M., Khalil, R.: Abel's formula and wronskian for conformable fractional differential equations. Int. J. Differ. Equ. Appl. 13(3), 177-183 (2014)

3. Agarwal, R.P., Dragomir, S.S.: An application of Hayashi's inequality for differentiable functions. Comput. Math. Appl. 32(6), 95-99 (1996)

4. Anderson, D.R.: Taylor's formula and integral inequalities for conformable fractional derivatives. arXiv:1409.5888 (2014)

5. Cerone, P., Dragomir, S.S.: On a weighted generalization of Iyengar type inequalities involving bounded first derivative. Math. Inequal. Appl. 3(1), 35-44 (2000)

6. Dragomir, S.S., Wang, S.: Applications of Iyengar's type inequalities to the estimation of error bounds for the trapezoidal quadrature rule. Tamkang J. Math. 29(1), 55-58 (1998)

7. Iyengar, K.S.K.: Note on an inequality. Math. Stud. 6, 75-76 (1938)

8. Khalil, R., Al Horani, M., Yousef, A., Sababheh, M.: A new definition of fractional derivative. J. Comput. Appl. Math. 264, 65-70 (2014)

9. Liu, Z.: On a trapezoidal type rule for weighted integrals. Tamkang J. Math. 35(3), 227-234 (2004)

10. Liu, W., Ngo, Q.-A.: Some Iyengar-type inequalities on time scales for functions whose second derivatives are bounded. Appl. Math. Comput. 216, 3244-3251 (2010)

11. Mitrinović, D.S., Pečarić, J.E., Fink, A.M.: Inequalities for Functions and Their Integrals and Derivatives. Kluwer, Dordrecht (1994)

12. Sarikaya, M.Z.: On weighted Iyengar type inequalities on time scales. Appl. Math. Lett. 22(9), 1340-1344 (2009) 